\title{
Underground Corrosion After 32 Years: A Study Of Fate And Transport, Annual Report For EMSP \#86803
}

\author{
M. K. Adler Flitton \\ June 2005
}

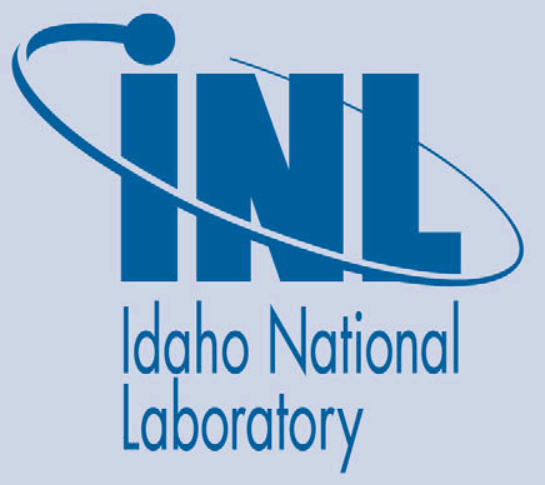

The INL is a U.S. Department of Energy National Laboratory operated by Battelle Energy Alliance 
INL/EXT-05-00379

\title{
Underground Corrosion After 32 Years: A Study Of Fate And Transport, Annual Report for EMSP \# 86803
}

\author{
M. K. Adler Flitton
}

June 2005

\author{
Idaho National Laboratory \\ Idaho Falls, Idaho 83415
}

Prepared for the

U.S. Department of Energy 


\section{Annual Report for EMSP \# 86803}

\section{Underground Corrosion after 32 Years: A Study of Fate and Transport}

Principal Investigator: M.K. Adler Flitton, Idaho National Laboratory, Idaho Falls, ID 83415, (208) 526-0525, mka@inel.gov.

\section{Research Objective}

Improved estimates for corrosion rates in variably saturated porous media are required by the U.S. Department of Energy to maintain long-term storage of radioactive contaminants in stainless steel containers. To better define these parameters, research was undertaken to complete the National Institute of Standards and Technology's (NIST) long-term study of buried stainless steel began 35 years ago. The 1970 study was initiated by the National Bureau of Standards (NBS), now known as NIST, when over 1000 specimens - including stainless steel Types 201, 202, 301, 304, 316, 409, 410, 430, and 434 - configured as plates, U-bend, and tubes in both annealed and cold worked conditions with various treatmentswere buried at six distinctive soil-type sites throughout the United States. During the first eight years of the study, four of five planned removals were completed with specimens retrieved after one, two, four, and eight years at each of the six sites. The fifth and final set of specimens remained undisturbed for over 34 years.

Researchers from Department of Energy (DOE) National Laboratories at the Idaho and the Savannah River Sites recovered and are analyzing part of a final set of specimens buried at Site D, near Wildwood, NJ. Findings included estimates for 32-year corrosion rates, transport of corrosion product, and elucidation of the site's hydrogeobiochemistry. An interdisciplinary research team unraveled the complicated interrelationships among metal integrity, corrosion rates, corrosion mechanisms, soil properties, soil microbiology, plant and animal interaction with corrosion products, and fate and transport of metallic ions. This research provides long-term corrosion and transport data (without the 35-year test expense) that can reduce the uncertainty associated with long-term waste storage and improve fate and transport modeling predictions throughout the DOE complex. The research also provides improvements in several characterization techniques.

\section{Research Progress and Implications} The objectives for this research are being accomplished through separate investigations in the different discipline areas that will be integrated to assess corrosion and the surrounding environmental factors. This report presents progress as of June 2005, the 3 rd year in a 4-year project. Although the individual investigations have yet to be integrated, their findings are reported below.

Site D specimen recovery, near Wildwood, N.J. A total of 53 stainless steel specimens were removed from one of the 1970 trenches in April 2004. The specimens were recovered about $3 \mathrm{ft}$ below land surface, spaced about 1 $\mathrm{ft}$ apart, from approximately $2 \mathrm{ft}$ below the water table (Figure 1). Roots were entwined around some specimens. (When originally buried, neither ground water nor plant roots were a concern.)

Site Environmental Investigations. An environmental monitoring station was established and data collected remotely. Findings indicate that water levels can routinely vary by as much as several feet with tidal fluctuations increasing/decreasing water levels. Results suggest that over the 35-year test duration, hydrological conditions have fluctuated resulting in the specimens being buried in a variably saturated zone. Chemical changes are also evident. Sands are evolving 
into soils coastal progression occurs and as trees and shrubs replace beach grasses and supply abundant organic material (Figure 2). The slightly acidic groundwater ( $\mathrm{pH} 4.7)$, high in iron and low in chromium and nickel, sustains localized reducing zones.

During the 35-year test duration, climate change has factored in the underground corrosion activity.

Specimen Mass Loss. Visual observations indicate that most specimens show little or no corrosion, regardless of type, treatment, or configuration. Most specimens were retrieved from the wet sand - shinny and clean (Figure

3 ). The notable exceptions were the sensitized Types 301 and Type 304 specimens.

Specimens were divided among various investigators where specimens and adhering material have been carefully examined. Specimens are being visually examined, cleaned, weighed, measured, and photographed - all leading to mass loss determinations. NIST personnel are assisting in providing a methodology to determine mass loss uncertainty. Results indicate that the sensitized specimens-Types 304 and Type 301 - sustained the greatest corrosion, whether U-bend or plate. To a lesser degree, the 400 series (Types 409, 410, and 434) evidenced local corrosion. Rust and black staining was observed on these specimens; sandy corrosion product adhered to localized areas; and pitting was evident. Most attacks occurred in localized areas as shown in Figure 4.

Stressed-specimen Investigation. The surfaces and cross sections of each U-bend specimen were examined in both stressed and unstressed areas, including the crevices created by the double U-bend specimens. Only sensitized Types 301 and 304 specimens exhibited significant corrosion. The sensitized and passivated, unwelded, half-hardened double U-bend Type 301 specimen fractured through the entire thickness of both U-bends in the stressed area (Figure 5). The fracture was intergranular and occurred along one side of the apex of the bends. The specimen was pitted on all surfaces, and significant pitting (average of 10 mils deep) was found close to the edges and also on the surfaces in the stressed areas. Shallower pits were found on the unstressed surfaces, away from the edges. Intergranular attack occurred in stressed and unstressed areas. When cleaned, the specimen separated along the fracture (Figure 6).

Examination of the sensitized and passivated, double U-bend Type 304 specimen, intergranular stress corrosion cracking was clearly evident in the stressed area on the top surface of the outer U-bend and also on the top surface (crevice side) of the inner U-bend.

Staining was obvious on the crevice side of both the inner and outer U-bend coupons. Cracks and pits were within the stained areas. A cross section through a cracked area showed that the coupon interior corroded away substantially, with very little indication of attack initiation on the surface of the coupons. Instead, the edges of coupons seemed to be the most likely starting point for corrosion.

Sensitization of these specimens prior to burial was most likely the reason for the extent of corrosion. The other stress specimens exhibited minor attack. Superficial intergranular attack (less than one mil deep) was seen on their surfaces.

Transport Investigations. One of the methods used to define the presence of corrosion product in the sand was Laser Desorption Mass Spectroscopy (LDMS).

Prior to looking for corrosion products, the background sand was well characterized using LDMS. (When compared against traditional methods, results suggest that the LDMS is an improved method for soil/ corrosion product characterization.) Three distinct types of sand make up the background soil: one white and two black. High potassium peaks dominate the white sand spectra. Spectra for both of the black sand types are high in iron but differ in concentrations of titanium (Figure 7). The percent abundance (ordinate) is determined by scaling the most plentiful component as $100 \%$ with all other peaks relative to it.

Corrosion product from specimens excavated intact in large boxes (with surrounding sand) (Figure $8 \mathrm{a}-\mathrm{f}$ ) was mapped in relation to the 
specimen. The sensitized Type 304 plate being recovered from the surrounding sand shows a plume of corrosion products emanating several inches into the surrounding soil. The LDMS spectrum differs when corrosion product is present-along with a high potassium spike indicative of white sand, there are elevated chromium and iron spikes (Figure 9). Iron and chromium are the largest chemical components of Type 304 stainless steel. Data suggest that corrosion product incorporates preferentially with the white sand as evidenced by the presence of the potassium peak.

Aqueous transport of either chromium or nickel was not detectable from groundwater samples analyzed.

Microbial Investigations. The objective of the microbiological component of this study was to demonstrate applicability of an innovative radioactive isotope method for imaging microbial activity to a comprehensive study of metal corrosion. The technique was combined with a more conventional radioactive tracer method for detecting sulfate reduction activity in soil associated with the specimens. Tests were performed on four of the stainless steel specimens (Figure 10) and soils recovered from the Wildwood, New Jersey site. Together the techniques provide a method for evaluating low metabolic levels of activity that have the potential for significant cumulative corrosion effects. The findings have direct relevance to material storage and waste disposal throughout the nuclear industry. Results from this study show the feasibility of mapping microbial activity to substrate surfaces for evaluation of corrosion potential. Soil organisms, initially present at very low numbers, may ultimately colonize resistant materials such as stainless steels. Results are described in detail in a peer-reviewed publication being prepared.

\section{Planned Activities}

- Integrate individual investigations to determine environmental impacts on corrosion and corrosion impacts on the environment.

- Thirty-two year corrosion rates will be estimated for each specimen.

- Corrosion product transport rates and distances will be determined.

- Determination on the viability of specimen welds will be finished.

- Determine corrosion effects for stressed specimens.

- Develop/calibrate numerical tool to simulate corrosion transport.

- Paper accepted to NACE 2006.

- Three peer-reviewed papers are being written or are in press.

\section{Information Access}

Adler Flitton, M.K. and L.R. Zirker, 2001, Digging for Treasure-Unique Fate and Transport Study, Waste Management, 2001. Tucson AZ., February 5-9, 2001.

Evans, Wm. and M.K. Adler Flitton, 2002, Underground Corrosion Rate and Transfer, INEEL symposium, July 7, 2002.

Adler Flitton, M.K., Long-term Underground Corrosion Investigation. EMSP Annual PI meeting, May 6-7, 2003, EMSL Facility, Richland, WA.

Bishop, C.W., M.K. Adler Flitton, J.R. Scott, M.E.Delwiche and K.M. Marshall, 2004, Underground Corrosion after 32 Years: A Study of Fate and Transport. American Geophysical Union Meeting, San Francisco, CA, Dec. 13-17, 2005.

Adler Flitton, M.K. and E. Escalante, 2003, "Simulated Service Testing in Soil," ASM Handbook, Vol. 13A Corrosion: fundamentals, Testing, and Protection. October 2003, Pg 497-500. 


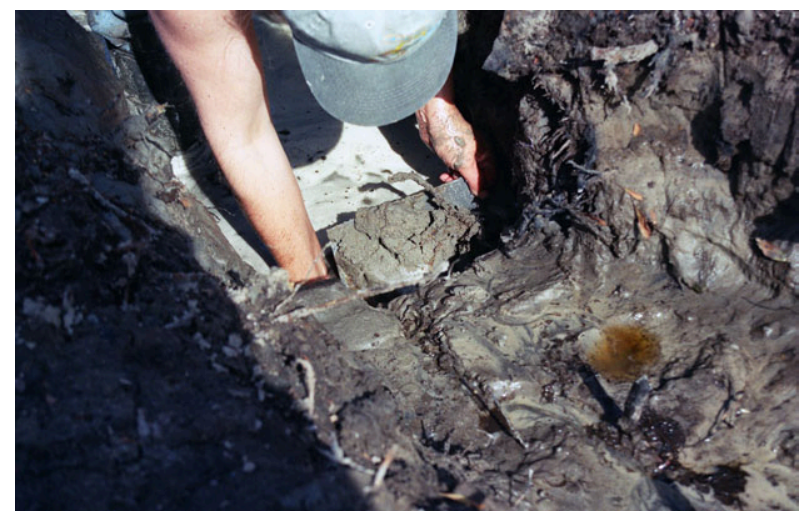

Figure 1. Specimen being recovered.

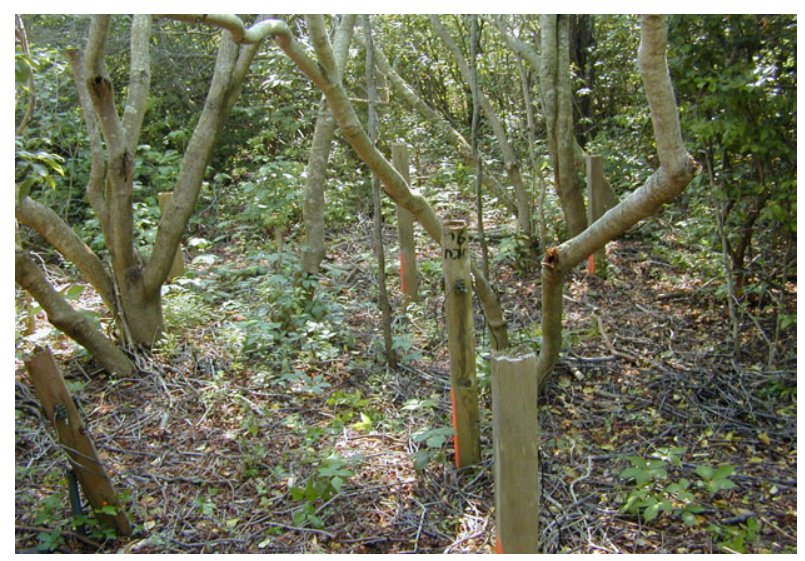

Figure 2. Sand and organic material support shrubs and medium sized trees.

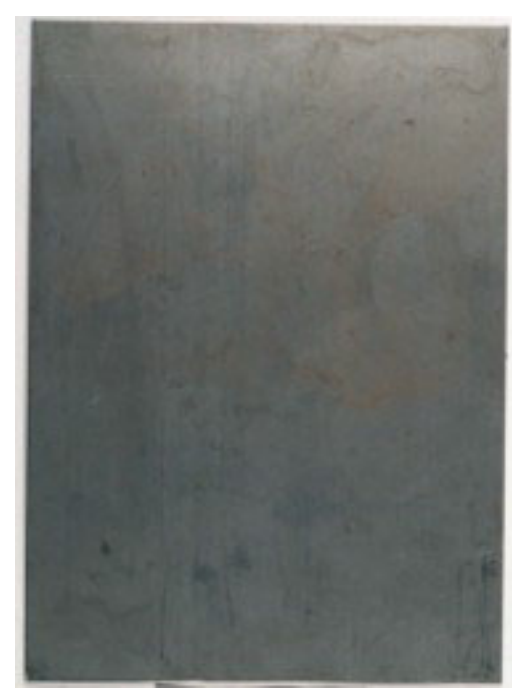

Figure 3. Annealed Type 316 plate with little visible corrosion.

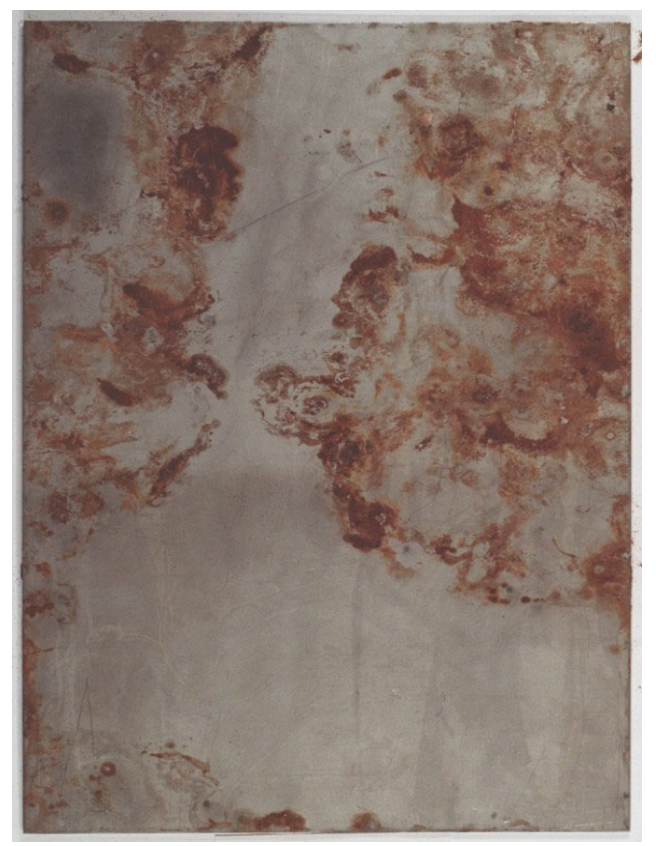

Figure 4. Corroded surface of Type 301 plate.

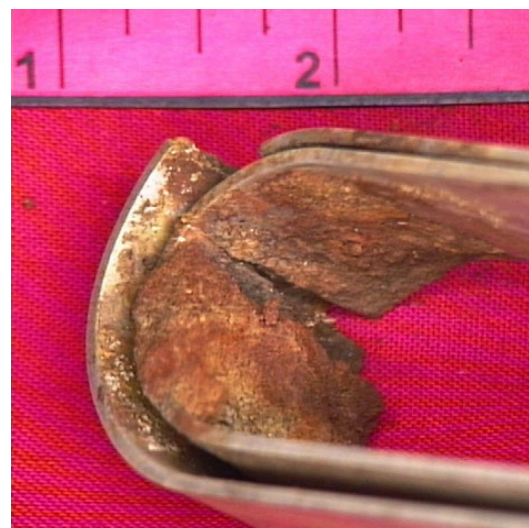

Figure 5.

Sensitized Type 301 spcimen showing stress corrosion cracks through stressed end.

Figure 5. Fractured stress corrosion specimen.

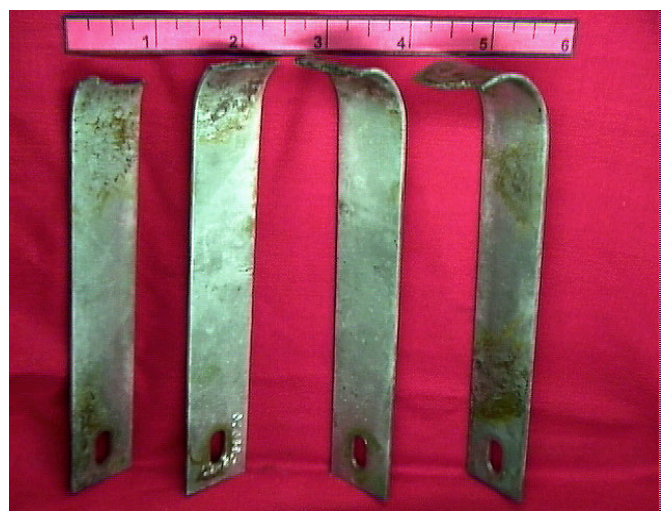

Figure 6. Cleaned stress corrosion specimen. 


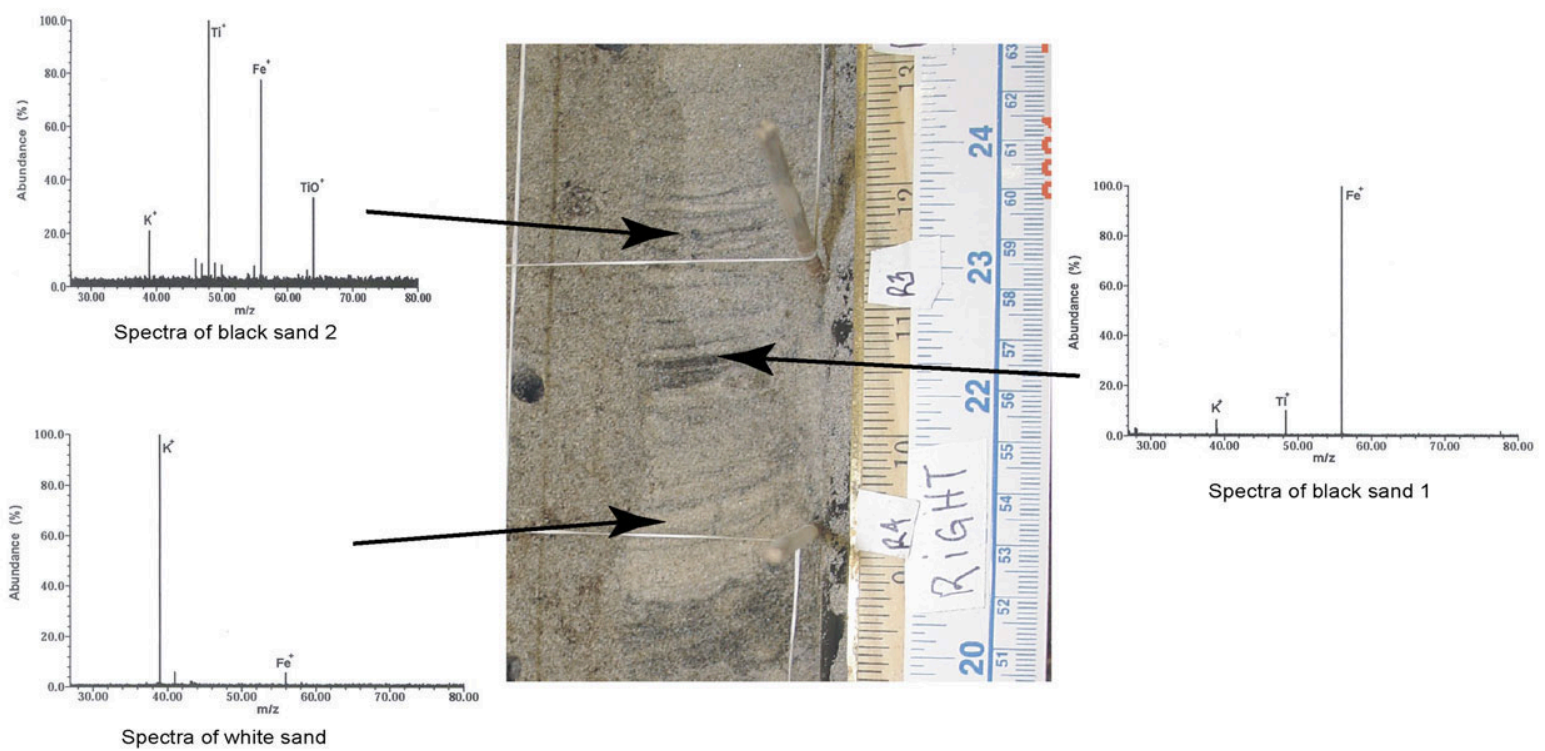

Figure 7. LDMS spectra of background sand types - 2 black and one white.

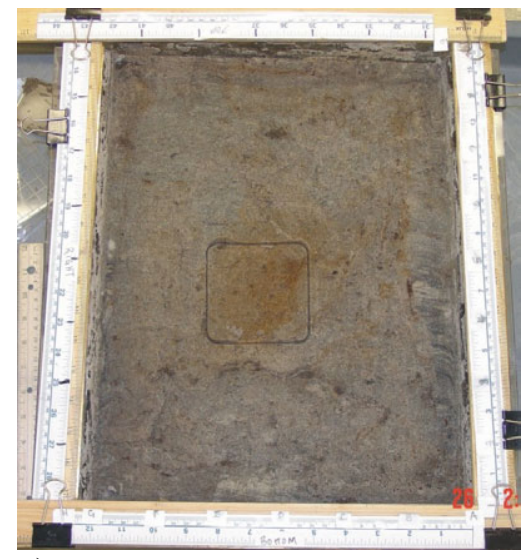

a)

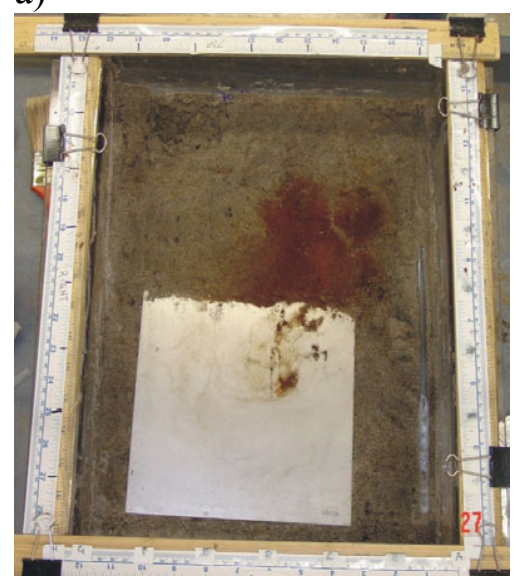

d)

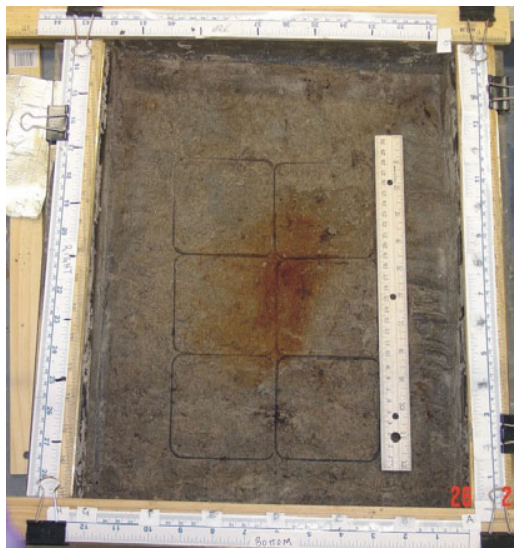

b)

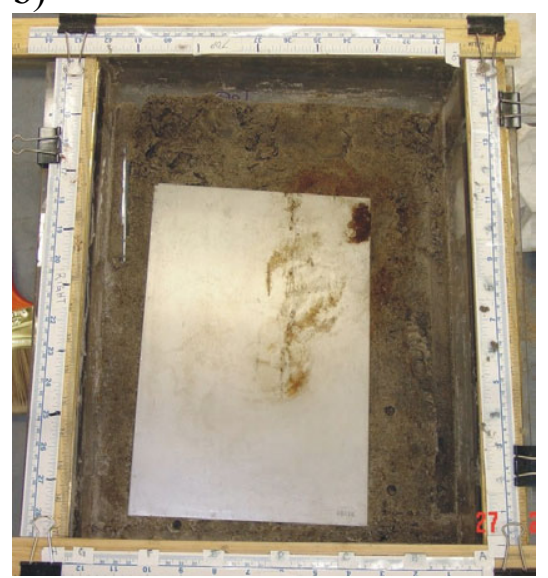

e)

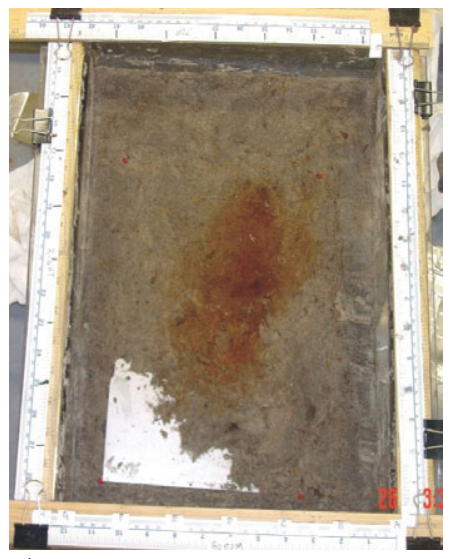

c)

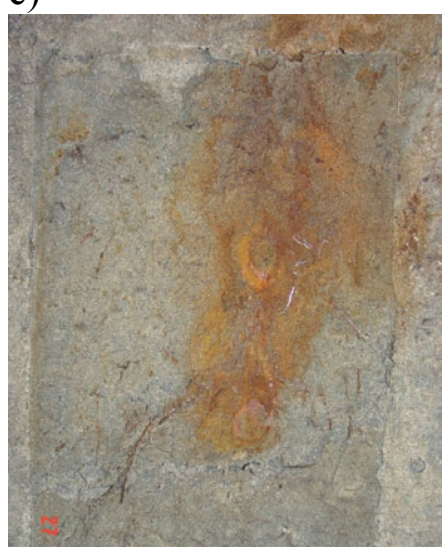

f)

Figure 8. Sensitized Type 304 specimen, recovered intact with surrounding sand, is shown above. Sand is mapped and sampled. Corrosion stain visible first in a) becomes darker closer to the specimen. Specimen is entirely visible in e) showing attack in upper right. Corrosion stained soil directly under plate is shown in $\mathrm{f}$ ). 


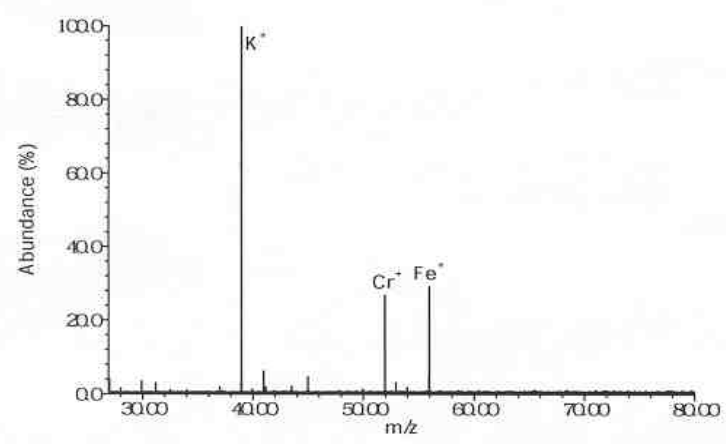

Figure 9. Spectrum of sand with the corrosion product. The abundant K indicates white sand and the elevated $\mathrm{Cr}$ and $\mathrm{Fe}$ are the corrosion products.

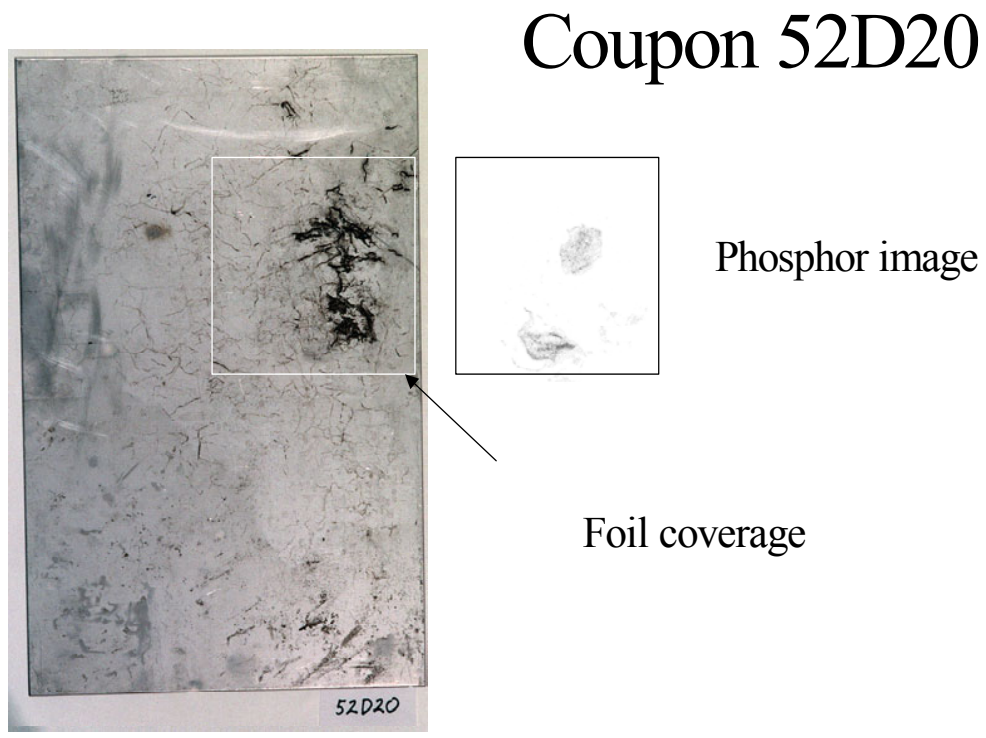

Figure 10. Silver foil was used to image sulfate reduction activity. Foils were placed in direct contact with coupons coated with radioactive labeled sulfate solution and with lactate. Sulfate reduction produced radioactive hydrogen sulfide that readily reacted with the foil surface to form silver sulfide. The radioactivity was recorded by phosphor imaging and mapped back to the original specimen surface. 